\title{
Treat the patient, not just the eye pressure
}

\author{
Dexter YL Leung, FRCS, FHKAM (Ophthalmology), Alvin KH Kwok *, MD, PhD \\ Department of Ophthalmology, Hong Kong Sanatorium and Hospital, Happy Valley, Hong Kong
}

Hong Kong Med J 2014;20:272-3

DOI: $10.12809 / \mathrm{hkmj} 144309$

Worldwide, glaucoma is regarded as one of the commonest causes of irreversible blindness. ${ }^{1}$ The global burden of glaucoma continues to rise: in 2010, at least 60.5 million people suffered from glaucoma, and by 2020, this is estimated to reach 79.6 million, of whom 11.2 million will be irreversibly blind in both eyes. ${ }^{1}$

Quality of life (QoL) includes dimensions such as physical health, mental health, general health perceptions, social functional status, and independence. $^{2}$ It has been shown that vision is consistently regarded as one of its key determinants. ${ }^{3}$ In a chronic disease such as glaucoma, the impact on vision, and hence QoL is, naturally, a very important subject.

Traditionally, research in glaucoma focuses on outcome parameters important for the ophthalmologists such as intra-ocular pressure (IOP), vertical cup-to-disc ratio (CDR), visual field status, and optical coherence tomography parameters such as the mean retinal nerve fibre layer (RNFL) thickness. No doubt these are important to the patients, but are no more than abstract ideas to them. Patients are more likely to be interested in their QoL. Unfortunately, some of the treatment modalities in glaucoma, while successful in preserving the optic nerve function, may sometimes have side-effects, diluting the gain in QoL. ${ }^{4}$ Even more unfortunately, in the past, few major ophthalmic clinical trials included QoL as part of their study protocol. So, a new treatment modality can be hugely successful in lowering IOP, preserving CDR, visual field, mean RNFL thickness and vision, and yet result in an unacceptable drop in QoL in the long term. In the year 2010, the US Food and Drug Administration endorsed that QoL assessment be included in all glaucoma clinical trials. ${ }^{5}$

Broadly speaking, there are currently four major categories of validated QoL analysis questionnaires used for glaucoma patients: the general healthrelated, vision-specific, glaucoma-specific, and utility value assessments. They differ in their internal consistency (as indicated by their Cronbach's alpha values), test-retest reliability, and correlation with severity of glaucoma. No matter what category of tools we choose, one unique challenge we face is that none of these validated QoL questionnaires is in Chinese (in fact, all are in English, thereby, creating a language barrier).
The study by Lee et $\mathrm{al}^{6}$ represents a nice attempt to address these issues. Firstly, the authors translated the Glaucoma Quality of Life-15 questionnaire (GQL-15) into traditional Chinese via a careful back-translation procedure. The questionnaire is a relatively easy-to-use, glaucoma-specific QoL tool addressing four aspects: (1) central and near vision; (2) peripheral vision; (3) dark adaptation and glare; and (4) outdoor mobility.

Secondly, they correlate this Chinese version of GQL-15 with a relatively new and important glaucoma clinical parameter, namely, the visual field index (VFI). First devised by Bengtsson and Heijl ${ }^{7}$ in 2008, VFI is automatically calculated using the newer Humphrey visual field analyser. Visual field index has been shown to reliably correlate with visual field loss from glaucoma, and be considerably less affected by visual loss as a result of concurrent cataract, which is also common in the same age-group of patients with glaucoma. ${ }^{7}$ Using VFI also allows the clinician to determine glaucoma disease progression (ie worsening) using a trend-based algorithm in addition to the traditional event-based algorithm. It has been demonstrated that trend-based determination of disease progression may be more robust than an event-based one, and incorporating both trend and event-based analyses can improve detection of glaucoma progression. ${ }^{8}$ Visual field index is increasingly being used in large clinical glaucoma trials and, clinically, is convenient to measure. The choice of studying the correlation between VFI and GQL-15 is a good way forward as currently there are few similar published data on this topic.

In this study, ${ }^{6}$ the authors found that a lower VFI correlated well with poorer GQL-15 scores and, hence, a lower QoL. The most problematic activities affecting QoL in these patients were "adjusting to bright lights", "going from a light to a dark room or vice versa", and "seeing at night". While these difficulties may seem immediately obvious to the eye doctors, the findings may have greater implications to the architectural lighting design specialists. It is high time now for Hong Kong to have a more mature discussion on the design of the city's exterior and interior lighting to facilitate the visually impaired citizens. Some of the suggested lighting engineering measures are, for example, plenty of floor lamps and table lamps in recreation and reading areas; usage of adjustable blinds, sheer curtains, or draperies for 
window coverings to allow adjustment of natural light; choice of brightly coloured vases and lamps near the furniture to make them easier to locate; elimination of hazards such as electrical cords in the pathway; avoiding waxing the floor; lighting the stairways clearly and uniformly; installation of grab bars; placing signs at eye level, with large lettering and braille signage according to guidelines, for example, from the Americans with Disabilities Act, etc. ${ }^{9}$ A concerted effort from all stakeholders of the society, from the Government to the architectural societies including the lighting specialists, to various blindness prevention organisations including the ophthalmologists, is needed to effect positive changes.

The ultimate goal of glaucoma treatment is to maximise QoL and patient satisfaction, not just creating a beautiful set of numerical figures in IOP/CDR/visual field/RNFL thickness. Addressing issues relating to QoL will allow both the clinicians and patients to re-orientate themselves towards a common realistic therapeutic programme, leading to a more harmonious partnership in care. So here is an old truth newly understood: we care for the patient as a human, not just for the parameters.

\section{References}

1. Quigley HA, Broman AT. The number of people with glaucoma worldwide in 2010 and 2020. Br J Ophthalmol 2006;90:262-7.

2. Felce D, Perry J. Quality of life: its definition and measurement. Res Dev Disabil 1995;16:51-74.

3. Spaeth G, Walt J, Keener J. Evaluation of quality of life for patients with glaucoma. Am J Ophthalmol 2006;141(1 Suppl):S3-14.

4. Leung DY, Tham CC. Management of bleb complications after trabeculectomy. Semin Ophthalmol 2013;28:14456.

5. Varma R, Richman EA, Ferris FL 3rd, Bressler NM. Use of patient-reported outcomes in medical product development: a report from the 2009 NEI/FDA Clinical Trial Endpoints Symposium. Invest Ophthalmol Vis Sci 2010;51:6095-103.

6. Lee JW, Chan CW, Chan JC, Li Q, Lai JS. The association between clinical parameters and glaucoma-specific quality of life in Chinese primary open-angle glaucoma patients. Hong Kong Med J 2014;20:274-8.

7. Bengtsson B, Heijl A. A visual field index for calculation of glaucoma rate of progression. Am J Ophthalmol 2008;145:343-53.

8. Medeiros FA, Weinreb RN, Moore G, Liebmann JM, Girkin CA, Zangwill LM. Integrating event- and trend-based analyses to improve detection of glaucomatous visual field progression. Ophthalmology 2012;119:458-67.

9. Information and Technical Assistance on the Americans with Disabilities Act, Civil Rights Division, United States Department of Justice. Available from: http://www.ada. gov/2010_regs.htm. Accessed 1 Jun 2014. 